\title{
Association Between Thrombospondin-1, Angiogenesis Related Markers, and Extracellular Matrix Components with Colorectal Cancer Outcome
}

\author{
Antigony Mitselou ${ }^{1, *}$, Dimitris L. Arvanitis ${ }^{2}$, Urania Skoufi $^{3}$, Dimitris Tsironis ${ }^{4}$, Evageli Lampri ${ }^{5}$, \\ Ioannis Nesseris ${ }^{5}$, Theodoros Vougiouklakis ${ }^{1}$, Evagelos Briassoulis ${ }^{6}$ and Elli Ioachim ${ }^{3}$ \\ ${ }^{1,5,6}$ Department of Forensic Pathology, Pathology and Oncology, University Hospital of Ioannina, Greece \\ ${ }^{2}$ Department of Anatomy, Medical School University of Thessalia, Greece \\ ${ }^{3,4}$ Department of Pathology and Surgery, General Hospital “G. Hatzikostas” Ioannina, Greece
}

\begin{abstract}
Background: Angiogenesis is a multistep process that depends on the balance of proangiogenic factors and inhibitors as well as on interactions with the extracellular matrix. Thrombospondin-1 (TSP-1) is an endogenous inhibitor of angiogenesis encoded by THBS1 gene, whose promoter is activated by $\mathrm{p} 53$.
\end{abstract}

Aim: To evaluate the relevance of TSP-1 in patients with colorectal cancer.

Material and Methods: We examined the immunohistochemical expression of angiogenic agents (VEGF and CD34), proliferation associated indices, extracellular matrix components (tenascin, fibronectin, laminin, and collagen type IV), and the antiangiogenic agent TPS-1 in 97 patients with colorectal carcinoma (CRC) and correlated their expression levels with clinicopathological parameters.

\begin{abstract}
Results: TSP-1 was detected in the tumor cells, stroma and perivascular tissue. High and moderate tumor TSP-1 expression was observed in $24.75 \%$, weak in $19.6 \%$, while $55.7 \%$ of the cases were negative. High stromal expression was observed in $40.2 \%$ and perivascular stain was noted in $31.95 \%$ of the cases. Stromal TSP-1 expression was correlated with tumor type and tumor grade $(\mathrm{p}=0.001$, and $\mathrm{p}=0.041$ respectively) and with $\mathrm{ECM}$ components expression: tenascin $(\mathrm{p}=0.053)$, fibronectin $(\mathrm{p}=0.063)$, collagen type IV $(\mathrm{p}=0.004)$ and laminin $(\mathrm{p}=0.0001)$. The relationship of TSP-1 expression with tumor angiogenesis, growth fraction, $\mathrm{p} 53$ protein expression, and overall survival was not significant.

Conclusions: Our data suggest that both tumor and stromal TSP-1 expression may not be a direct antiangiogenic factor, although it seems to be implicated in the remodeling of colorectal cancer tissue through interaction with other extracellular matrix components.
\end{abstract}

Keywords: Thrombospondin-1, VEGF, CD34, tenascin, fibronectin, laminin, type IV collagen, colorectal cancer.

\section{INTRODUCTION}

Colorectal cancer (CRC) is a leading cause of cancer morbidity and mortality in economically advanced regions of the world. It is the third most frequently diagnosed visceral cancer and the second leading cause of cancer mortality accounting for about $10 \%$ of all cancer deaths in both genders combined [1,2]. Variables that have been found to have prognostic value include tumour stage, grade and the presence of vascular invasion. Hematogenous and nodal metastasis, are the major causes of death in patients with CRC [3]. Approximately $10-30 \%$ of colon tumours will recur despite the absence of lymph node metastases at the time of resection [1-3]. The progressive growth of colonic cancer and the subsequent metastatic process is dependent on an angiogenic network.

*Address correspondence to this author at the Department of Forensic Pathology, Medical School, University of Ioannina, Ioannina, Greece;

Tel: +302651007586; Fax: +302651007857; E-mail: amitselo@cc.uoi.gr
Angiogenesis is a complex multistep dynamic process, involving extravasation of plasma proteins, degradation of extracellular matrix (ECM), endothelial cell proliferation, migration, and differentiation into capillaries [4]. The development of new blood vessels within a tumour depends upon the balance between angiogenic and antiangiogenic factors. These factors may be produced by the tumour cells themselves or by associated stromal and inflammatory cells. Thrombospondin-1 (TSP-1) is thought to be an antiangiogenic factor $[5,6]$. The TSP is a family of glycoproteins that induce platelet aggregation and inhibit angiogenesis $[5,6]$. Of the five TSP family members identified to date, TSP-1 and TSP-2 were shown to be inhibitory factors on angiogenesis due to the presence of procollagen homology region and type I repeats [6].

The pathways controlling the switch to angiogenic phenotype in tumours are complex and poorly characterized. There is evidence that changes in oncogene and tumour suppressor gene expression influence new vessel growth during tumour progression [7]. There is a close relationship between 
Table 1. Patient's Characteristics in the Present Study

\begin{tabular}{|c|c|c|}
\hline Variable & Patients (n) & $\%$ \\
\hline \multicolumn{3}{|l|}{ Sex } \\
\hline Male & 53 & 54.6 \\
\hline Female & 44 & 45.4 \\
\hline \multicolumn{3}{|l|}{ Age } \\
\hline$<60$ years & 34 & 35.05 \\
\hline$\geq 60$ years & 63 & 64.94 \\
\hline \multicolumn{3}{|l|}{ Grading } \\
\hline G1 & 19 & 19.58 \\
\hline G2 & 67 & 69.07 \\
\hline G3 & 11 & 11.34 \\
\hline \multicolumn{3}{|l|}{ Dukes' Stage } \\
\hline B & 56 & 57.73 \\
\hline $\mathrm{C}$ & 41 & 42.26 \\
\hline \multicolumn{3}{|l|}{ Tumor Size } \\
\hline$\leq 5 \mathrm{~cm}$ & 39 & 40.20 \\
\hline$>5 \mathrm{~cm}$ & 58 & 59.79 \\
\hline \multicolumn{3}{|l|}{ Tumor Type } \\
\hline Mucinus & 21 & 21.64 \\
\hline Non-mucinus & 76 & 78.35 \\
\hline
\end{tabular}

the expression of TSP-1 and wild-type p53, in the manner that loss of wild-type p53 being associated with loss of TSP1 expression [8,9]. Decreased expression of the TSP-1 is correlated with metastatic potential in prostate cancer [10], non-small lung cancer [11], breast cancer [12], and bladder cancer [13]. In CRC, TSP-1 has been shown to have both an angiogenic effect $[14,15]$ and conversely be associated with venous invasion and tumour progression [16].

Vascular endothelial growth factor (VEGF), is a mitogen for endothelial cells, and may be a crucial mediator for neovascularization in human tumours [17], and its expression has been proposed to be predictive of the clinical outcome in terms of cancer metastasis $[18,19]$. Tumour vascularization can be also examined immunohistochemically using CD34 antibody to determine the microvessel density (MVD). The grade of MVD in tumours is considered to be a measure of angiogenesis and a strong prognosticator in patients with a variety of cancers [20].

Stromal components also play a critical and often underappreciated role in the formation of vascular stroma through the regulation of functions such as cell adhesion, migration, and gene expression by controlling the availability of growth factors [21]. Tenascin (TN) and fibronectin (FN) are glycoprotein components of the extracellular matrix (ECM) which seems to have competitive functions. It can be speculated that this competitive relationship between these molecules is important for cellular functions [22]. In infiltrating CRC, TN is expressed in the stroma and appears to be associated with progressive disease and poor prognosis [23]. FN is regarded as the major mesenchymal ECM glycoprotein involved in cell-matrix and cell-cell adhesion, cell migration, morphogenesis, differentiation, and oncogene transformation [24]. In CRC, FN immunostain shown a strong expression and different distribution compared with normal colorectal tissue $[23,24]$. Collagen type IV (Coll IV), constitute the major intrinsic component of basement membrane $(\mathrm{BM})$ and is involved in cellular adhesion to BM and the extracellular matrix [25]. Coll IV degradation products play an important role during angiogenesis, tissue remodelling, and cancer progression [25]. Loss of a well-defined basement membrane is one of the features of neoplastic proliferation for epithelialderived tumors. Laminin (LN), a major glycoprotein of BM which has been shown to regulate a variety of biological phenomena including cell attachment, growth, morphology, and cell migration by specific high affinity receptors, and plays an important role in the interaction of tumor cells with the BM, the differentiation, invasion and metastasis of tumors [26].

Our study was intended to (a) evaluate the patterns of TSP-1 expression in tumour cells and tumour stroma, in patients with $\mathrm{CRC}$; its possible correlation with clinicopathological features such as: tumour size, tumour type, the grade of differentiation, vessels invasion, and lymph node involvement (Table 1); (b) investigate the relationship between tumour cell and stroma TSP-1 expression, and possible correlation with angiogenesis related factors: MVD and VEGF expression; (c) evaluate the relationship between TSP-1 expression to other biological parameters including proliferation indices (MIB1 and PCNA) and p53 protein; (d) investigate the correlation of the expression of TSP-1 with the ECM components: TN, FN, Coll IV, and LN; and (e) evaluate the prognostic significance of TSP-1 in CRC.

\section{MATERIALS AND METHODS}

The study population consisted of 97 patients whose tumors were completely removed surgically at the University Hospital of Ioannina, Greece. The patients included in this study had no other cancer. There were 53 (54.6\%) men and $44(45.4 \%)$ women. Sixty-three were $\geq 60$ years old and 34 were $<60$ years old. The mean age at diagnosis was 64.92 years (range $=26-86$ years). None of the patients had received chemotherapy or radiation therapy prior to surgery. The pathologic features were classified using the UICCTNM classification [27]. Tumors were graded according to the WHO classification criteria as well, moderately or poorly differentiated carcinomas [28]. When more than $50 \%$ of the tumor volume was mucin, the tumor was defined as mucinous carcinoma. Histopathological diagnosis was made routinely at the Department of Pathology of Ioannina University Hospital. All routine sections were carefully investigated to identify venous or lymphatic invasion. The largest diameter of the tumor was defined as the tumor size. The extent of tumor invasion/metastasis was based on the Astler and Coller modification of Dukes' classification system [27, 28]. Dukes' A cases were those in which the growth was confined to the submucosa of the colorectal wall and these were 
not included in this study. In Dukes' B cases, the growth spread by direct continuity into the extracolorectal tissues, but the lymph nodes were free from metastases. Dukes' C cases were those in which lymph node metastases were found $[27,28]$. The mean observation time was 4.7 years (range $=0.5-13.5$ years), of these patients, $33.7 \%$ had recurrence or distant metastases and $31.7 \%$ were dead from the disease or other causes after 5 years of follow-up.

The specimens were fixed in $10 \%$ neutral-buffered formaldehyde and embedded in paraffin. Based on the quality of the morphologic preservation of all available hematoxylin and eosin stained slides of the surgical specimen sections, we selected one or two paraffin blocks for each case. Consecutive $4 \mu \mathrm{m}$ sections were cut again from each study block: these sections were immunostained for TSP-1, tenascin, fibronectin, collagen type IV, laminin, Ki-67, PCNA, p53, VEGF, and CD34. In brief, tissue sections were deparaffinized in xylene and dehydrated. For the detection of thrombospondin-1 (DBS; dilution 1:30), Ki-67 (Dako; dilution 1:10), PCNA (Dako; dilution 1:20), p53 Dako; dilution 1:50), VEGF (Neomarker; dilution 1:50) and CD34 (Novo Castra; dilution 1:50) slides were immersed in citrate buffer $(0.1 \mathrm{~m}, \mathrm{pH} 0.6)$ in plastic Coplin jars and subjected to microwave irradiation twice for $15 \mathrm{~min}$. For the detection of TN (Dako; dilution 1:50), FN (Novo Castra; dilution 1:100), LN Menarin; dilution 1:1000), and Coll IV (Dako; dilution 1:50) slides were pre-treated with $1 \mu \mathrm{l} / \mathrm{ml}$ pronase (Dako) for 10 min at room temperature. Subsequently, all sections were treated for $30 \mathrm{~min}$ with $0.3 \%$ hydrogen peroxide in methanol to quench endogenous peroxidase activity and then incubated with primary antibodies. We used the method involving the streptavidin-biotin-peroxidase complex and developed the chromogen with immersion of the slides in a diaminobenzidine $\mathrm{H}_{2} \mathrm{O}_{2}$ substrate for $5 \mathrm{~min}$. The slides were counterstained in Harri's haematoxylin, dehydrated and mounted. To access the specificity of the reaction, control specimens were prepared from normal mucosa tissue. The antibodies used, sources and dilutions are shown in Table 2.

\section{Immunohistochemical Evaluation}

\section{Thrombospondin-1 and VEGF}

To evaluate the expression of TSP-1 and VEGF proteins, we established a combined score, corresponding to the sum of both: (a) staining intensity ( $0=$ negative, $1=$ weak, $2=$ moderate, $3=$ strong, $4=$ very strong staining) and (b) the staining extensive, percentage of positive cells $(0=0 \%, 1=1$ $25 \%, 2=26-50 \%, 3=51-75 \%, 4=76-100 \%$ ). The sum of both quantitative and qualitative immunostaining reached a maximum score of 8 . The combined scores were then divided into four main groups: $(-)=$ no immunostaining, score $0 ;(+)$ $=$ weak immunostaining score $1-2 ;(++)=$ moderate immunostaining, score $3-4 ;(+++)=$ strong immunostaining, score $5-8$. The immunostaining was assessed from numerically coded slides without any knowledge of histopathologic features, survival or other clinical data.

\section{Ki-67 (MIB-1) and PCNA}

Nuclear staining for Ki-67 and PCNA was calculated as the percentage of positive tumor cells in relation to the total number of cells encountered at least 5-10 representative high power fields (500-1000 epithelial cells). Every stained cell was considered positive. Each sample was first scanned at low magnification and at least 10 fields were assessed a high power examination.

\section{P53 Protein}

Nuclear staining for p53 was calculated as the percentage of the positive epithelial cell in relation with the total number of neoplastic cells. Every stained cell was considered positive irrespective of intensity. Each sample was first examined on low magnification and at least 5 representative fields were assessed with a high power magnification.

\section{Microvessel Count}

The criteria that we used for microvessel recognition were the same as used in previous studies [29, 30]. Briefly, as microvessels we considered individual or clusters of cells with or without lumens, positively stained by anti-CD34.

Table 2. Antibodies Used in the Current Study

\begin{tabular}{|c|c|c|c|}
\hline Antibodies & Supplier (Country) & Dilution & Incubation Time \\
\hline TSP-1 (A6.1) & DBS (UK) & $1: 30$ & $30 \mathrm{~min}$ \\
\hline CD34(QBEnd/10) & Novo Castra (UK) & $1: 50$ & 1 hour \\
\hline Tenascin (TN2) & Dako (Denmark) & $1: 50$ & 1 hour \\
\hline Collagen IV (clone, CIV22) & Dako (Denmark) & $1: 50$ & 1 hour \\
\hline P53 (DO-7) & Dako (Denmark) & 1: 50 & 1 hour \\
\hline Ki-67 (MIB1) & Dako (Denmark) & $1: 10$ & 1 hour \\
\hline
\end{tabular}


The lumens diameter had to be smaller than approximately eight red blood cells. Areas of fibrosis, necrosis, inflammation, and vessels with muscle wall, were excluded from counting. In each tumor, the three areas with highest vascularization ('hot spot') were selected. Individual microvessel counts were then made on a 20X 10 first and then 40X magnification. The mean microvessel density (MVD) obtained from the five fields was used for analysis. The immunoreactivity was interpreted by means of light-microscopic examination and evaluated independently by two pathologists (AM, EI). Differences in interpretation were reconciled by re-review of slides separately or jointly at a double-headed microscope. The staining was evaluated only in the areas with well-preserved tissue morphology and away from necrosis or artifacts.

\section{Extracellular Matrix Components}

Tumors were classified as "positive" when there was unequivocal immunostaining for TN, FN, Col IV, and LM of the matrix components in at least one representative area of the tumor. The positive tumors were semi-quantitatively scored as $1+, 2+$, and $3+$ corresponding to weak, moderate, and extensive immunoreactivity, respectively.

\section{STATISTICAL ANALYSIS}

All data were entered into a microcomputer and chisquare $\left(\mathrm{x}^{2}\right)$, univariate and multivariate analysis for variance were adopted in a Statistical Package for the Social Sciences (SPSS) 10.0 for windows program. Significant differences between the immunohistochemical expressions of markers were compared by the t-test for paired or non-paired values or ANOVA test if the data wee normally distributed. Correlation between these proteins was computed using the Pearson's correlation coefficient for normally distributed data or the Kendall's tau-b rank correlation coefficient where the data did not show a normal distribution. The prognostic significance of TSP-1 expression, in determining the risk of recurrence, was studied with both univariate (log rank test) and multivariate (Cox proportional hazards) ways of analysis, separately for each group of patients. P-values $\leq 0.05$ were considered statistically significant.

\section{RESULTS}

\section{TSP-1 Expression}

TSP-1 expression was detected in the cytoplasm of carcinoma cells (Fig. 1), in tumor stroma (Fig. 2), and in perivascular tissue. In tumor cells, TSP-1 expression was observed in $24 / 97(24.75 \%)$ of the cells with moderate to strong immunostaining, while 19/97 (19.6\%) were weak and 54/97 $(55.7 \%)$ of the cases were completely negative. In $7 / 97$ (7.2\%) cases, both expressions tumoral and stromal observed. 31/97 (31.95\%) tumor specimens expressed perivascular TSP-1 and 39/97 (40.2\%) expressed stromal TSP-1. In $58 / 97(59.8 \%)$ tumors, no stromal or perivascular TSP-1 expression was observed. In eleven tumors $(11.3 \%)$, both pattern of expression, stromal and perivascular, were observed. Statistically positive relationship was found between stromal TSP-1 expression and tumor cell TSP-1 expression $(\mathrm{p}=0.001)$, as well with perivascular TSP-1 expression $(p<0.0001)$. A positive correlation between stromal TSP-1

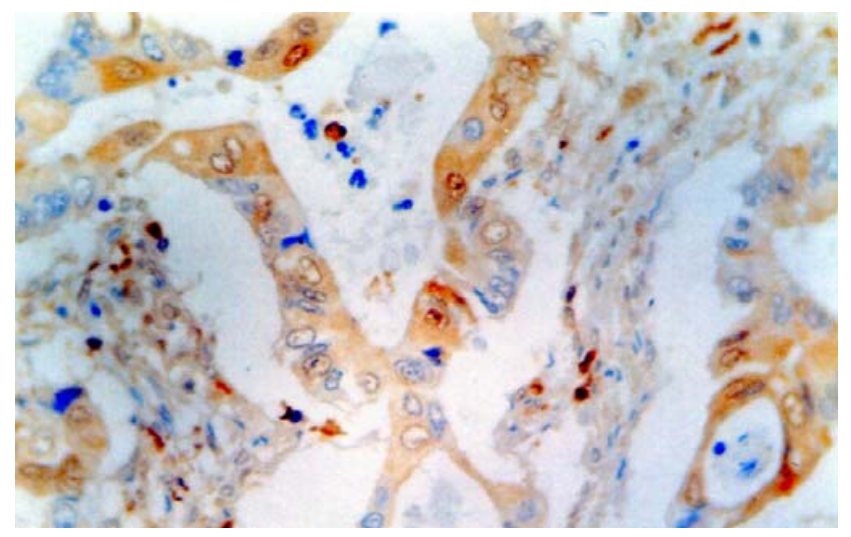

Fig. (1). Immunostaining of TSP-1 in tumor cells of moderate adenocarcinoma of colon (X400).

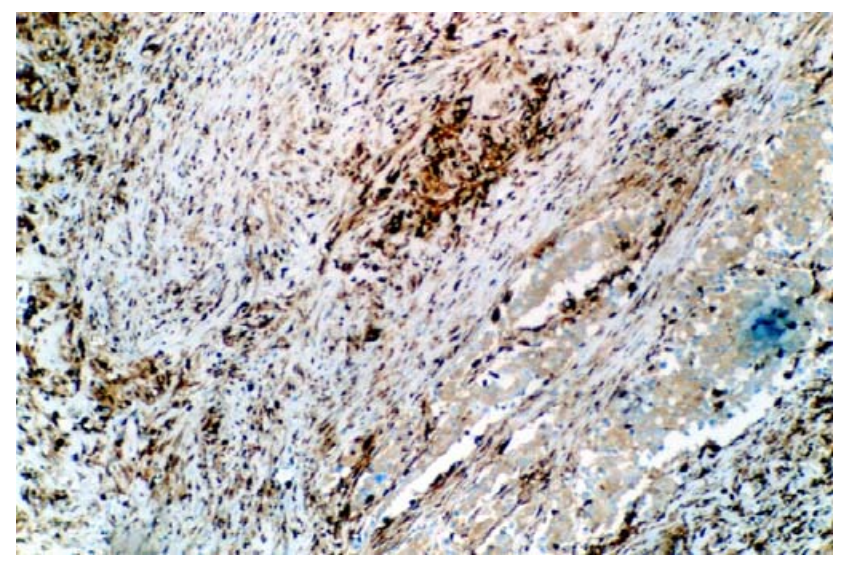

Fig. (2). Strong immunoreactivity of TSP-1 in the stroma of CRC (X200).

expression and the type of carcinoma (mucinous) and the grade of cancer ( $p=0.001$ and $p=0.041$ respectively) was observed. TSP-1 expression did not correlate with gender and size of the tumor (Table 3). Tumor, stromal, and perivascular TSP-1 expression did no correlate with p53 status, proliferative indices, and the other clinicopathological features.

\section{VEGF and MVD Expression}

VEGF immunohistochemical expression was detected in cytoplasm and in some cases in the membrane of carcinoma cells, although with different percentages of stained cells. Specifically 66 of the $97(68.1 \%)$ cases were strongly positive, $17(17.5 \%)$ were moderately positive, 5/97 (5.2\%) were weakly positive, and 9/97 (9.3\%) were negative. Strong immunoreactivity in the tumor vessels was observed in 43 $(44.3 \%)$ cases, moderately in $17 / 97(17.5 \%)$, weak in 23 $(23.7 \%)$ and $14 / 97(14.5 \%)$ cases were totally negative. Statistically no relationship was found between perivascular TSP-1 expression and VEGF vessels expression $(\mathrm{p}=0.082)$. CD34 immunoreactivity was detected in vascular endothelial cells. At the tumor site, the MVD ranged from 15 to 122 (mean \pm SD $56.3 \pm 21.3$ ). A positive association was observed between VEGF expression and MVD $(\mathrm{p}=0.0016)$, but we failed to demonstrate any correlation between MVD and TSP-1 tumoral or stromal expression (Table 4). 
Table 3. Thrombospondin-1 Expression in Correlation with Clinicopathological Data in Colorectal Cancer

\begin{tabular}{|c|c|c|c|c|c|}
\hline & \multicolumn{2}{|c|}{ TSP-1 Expression in Tumor Cells } & \multicolumn{2}{|c|}{ TSP-1 Expression in Tumor Stroma } & P Value \\
\hline \multicolumn{6}{|l|}{ Type } \\
\hline Nonmucinous & 67 & 10 & 59 & 18 & $p=0.001$ \\
\hline Mucinous & 14 & 6 & 9 & 11 & NS \\
\hline \multicolumn{6}{|l|}{ Size } \\
\hline$\leq 5 \mathrm{~cm}$ & 19 & 3 & 17 & 5 & NS \\
\hline$>5 \mathrm{~cm}$ & 23 & 3 & 19 & 7 & NS \\
\hline \multicolumn{6}{|l|}{ Grade } \\
\hline 1 & 12 & 4 & 12 & 2 & NS \\
\hline \multicolumn{6}{|l|}{ Duke's stage } \\
\hline $\mathrm{B}$ & 39 & 7 & 31 & 12 & NS \\
\hline $\mathrm{C}$ & 28 & 5 & 26 & 8 & NS \\
\hline \multicolumn{6}{|l|}{ Vessel Invasion } \\
\hline Negative & 69 & 15 & 61 & 24 & NS \\
\hline Positive & 11 & 2 & 9 & 3 & NS \\
\hline \multicolumn{6}{|l|}{ Ki67 } \\
\hline \multicolumn{6}{|l|}{ P53 } \\
\hline$<5 \%$ & 29 & 7 & 28 & 9 & $N S$ \\
\hline$\geq 5 \%$ & 49 & 11 & 39 & 19 & NS \\
\hline
\end{tabular}

Percentage of positive tumour cells

\section{Coll IV Expression}

A strong and well-defined collagen type IV staining is seen in the basement membrane of normal mucosal epithelium and around blood vessel walls. The epithelial cells themselves showed negative immune-reaction. Limited or lack of Coll IV staining was seen in 24 (24.7\%) of the cases, moderate in $31(31.9 \%)$ and strong in $25(25.8 \%)$ of the cases, while $17(17.5 \%)$ of the cases were negative (Fig. 3). In addition, Coll IV staining disappeared in poorly differentiated adenocarcinomas. A statistically significant association was found between Coll IV expression and stromal TSP1 expression $(\mathrm{p}=0.004)$.

\section{LN Expression}

Moderate and strong LN expression was observed in 71 (73.2\%) cases of well- and moderately differentiated CRCs, with continuous membrane LN staining. Weak or absent expression was noted in $26(26.8 \%)$ cases. Patches of LN immune-reaction was scattered throughout the tumor, mainly in poorly differentiated adenocarcinomas. When the staining patterns of poorly differentiated tumors were compared statistically with those of well- and moderately differentiated tumors, a significant difference was found $(\mathrm{p}<0.01)$. A statistically significant association between TSP-1 stromal expression and LN expression ( $\mathrm{p}=0.0001)$ was observed. 
Table 4. Thrombospondin-1 Expression in Correlation with Angiogenesis Related Markers and Extracellular Matrix Components in Colorectal Cancer

\begin{tabular}{|c|c|c|c|c|c|}
\hline & \multicolumn{2}{|c|}{ TSP-1 Expression in Tumor Cells } & \multicolumn{2}{|c|}{ TSP-1 Expression in Tumor Stroma } & \multirow[t]{2}{*}{ P Value } \\
\hline & + & ++ &,+++ & +++ & \\
\hline \multicolumn{6}{|l|}{ MVD } \\
\hline Low & 25 & 5 & 23 & 7 & NS \\
\hline High & 41 & 9 & 34 & 16 & NS \\
\hline \multicolumn{6}{|l|}{ VEGF } \\
\hline+ & 10 & 2 & 9 & 3 & NS \\
\hline++ & 4 & 3 & 5 & 2 & NS \\
\hline+++ & 57 & 9 & 48 & 18 & NS \\
\hline \multicolumn{6}{|l|}{ Tenascin } \\
\hline+ & 19 & 5 & 17 & 7 & NS \\
\hline++ & 26 & 6 & 21 & 11 & NS \\
\hline+++ & 25 & 2 & 21 & 6 & $\mathrm{P}=0.053$ \\
\hline \multicolumn{6}{|l|}{ Colagen IV } \\
\hline+ & 29 & 5 & 26 & 8 & NS \\
\hline++ & 24 & 5 & 22 & 7 & NS \\
\hline+++ & 20 & 3 & 14 & 9 & $\mathrm{P}=0.004$ \\
\hline \multicolumn{6}{|l|}{ Fibronectin } \\
\hline- & 4 & 2 & 5 & 1 & NS \\
\hline+ & 26 & 3 & 24 & 5 & NS \\
\hline++ & 42 & 8 & 32 & 18 & $\mathrm{P}=0.063$ \\
\hline \multicolumn{6}{|l|}{ Laminin } \\
\hline+ & 17 & 8 & 5 & 1 & NS \\
\hline++ & 24 & 6 & 6 & 11 & NS \\
\hline+++ & 19 & 7 & 47 & 12 & $\mathrm{P}=0.0001$ \\
\hline
\end{tabular}

\section{TN Expression}

TN was found mainly in the stroma surrounding malignant cells, while tumor epithelial cells were negative. The staining was weak and occasional in 17 (17.6\%) cases, moderate in $47(48.45 \%)$ cases, and strong in $23(23.71 \%)$ specimens. A significant statistically difference was found $(p<0.001)$ between staining pattern for poorly differentiated tumors when compared with well and moderately differentiated tumors. A borderline statistical relation was found between TSP-1 stromal and TN expression $(\mathrm{p}=0.053)$.

\section{FN Expression}

In CRCs, FN immunostaining showed positive reaction in the tumor stroma with a varying intensity, with orientation along connective tissue fibers as diffuse staining (Fig. 4). The staining was absent or weak in 8/97 (8.21\%) of the cases, mostly poorly differentiated adenocarcinomas, and mod- erate to strong in 89 (91.8\%) specimens of well- and moderately differentiated tumors (Fig. 4). A positive statistical re-

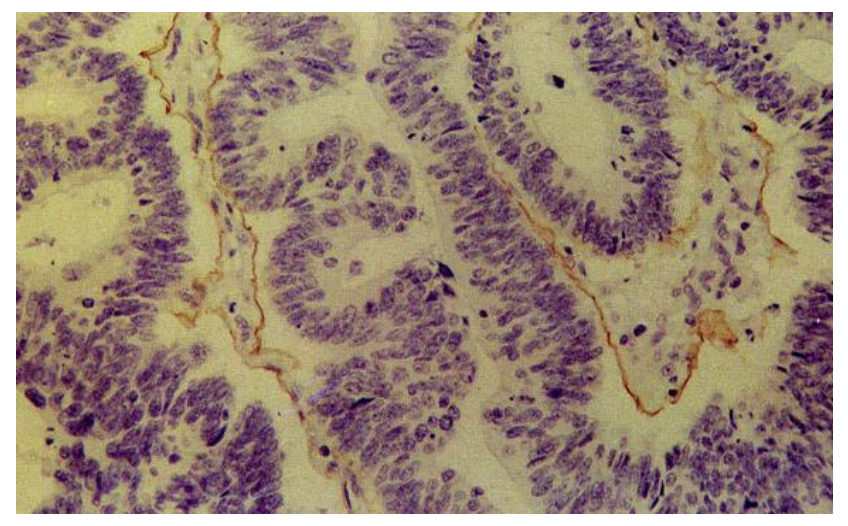

Fig. (3). Collagen type IV immunostain in CRC (X200). 


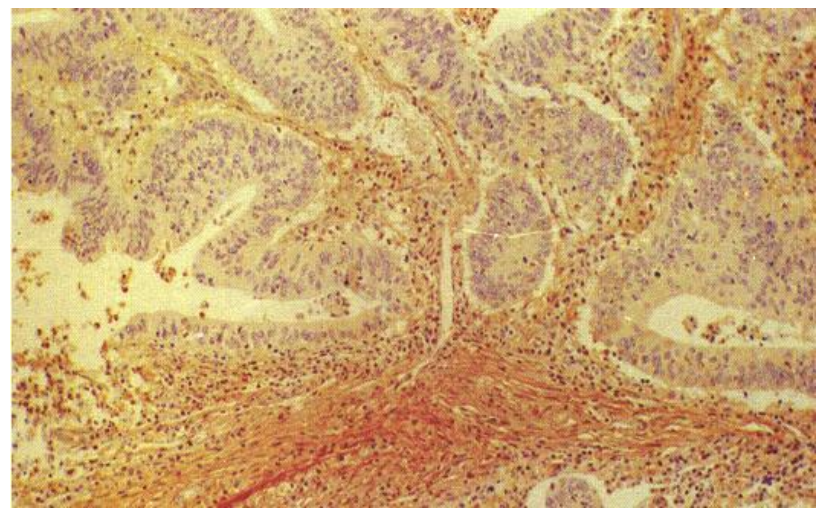

Fig. (4). Fibronectin immunoexpression in CRC (X100).

lation was found between TSP-1 stromal expression and FN expression $(\mathrm{p}=0.063)$.

The prognostic significance of TSP-1 expression in colorectal carcinomas in both tumor cells and tumor stroma in determining the risk of recurrence and progression with univariate and multivariate methods of analysis showed no statistically significant differences $(p=0.072$ and $p=0.076$ respectively).

\section{DISCUSSION}

Thrombospondin-1 is one of the strongest negative regulating molecules during neovascularization in tumors [5, 6]. It regulates the growth metastasis and angiogenesis in various tumors and is significant in predicting tumor malignancy. Some researches in their studies found that TSP-1 was distributed in the cytoplasm of tumor cells in thyroid cancer [31], breast cancer [32], hepatocellular carcinoma [33], gastric carcinoma [34], and CRC [19]; while others studies showed that TSP-1 was mainly expressed in the ECM in pancreatic cancer [35], CRC [16], esophageal squamous cell carcinoma [36], non-small cell lung cancer [10], ductal in situ carcinoma of the breast [37]. However, others studies reported TSP-1 expression in both cytoplasm and stroma, as well in CRC [9], bladder cancer [13], and breast cancer [38]. Thus, the expression mode of TSP-1 in tumors could vary in different tissues or organs. In the present study we found TSP-1 strong expression in the cytoplasm of tumor cells in $24.75 \%$ of the cases, $40.21 \%$ of cases showed immunostaining in the stroma, and $31.95 \%$ of the cases showed positivity in perivascular tissue, in accordance with the study of Tokunaga et al. [9]. These results may be due to the difference in TSP-1 products produced by tumor cells or to different antibodies used. Rojas et al, suggested that the tumor cells are a major source of TSP-1 and that methylation of TSP-1 substantially reduces TSP-1 activity in the tumors of the colon [39].

Different results have been reported in studies evaluating the correlation between TSP-1 expression and tumor stage and tumor grade. Some studies did not find a significant relationship between TSP-1 expression and stage and grade [14, 15], while other study found that decreased expression of TSP-1 was correlated with $\mathrm{T}$ and $\mathrm{N}$ grades with borderline significance [9]. In our study, we observed a statistically significant relationship between TSP-1 stromal expression and the type of cancer $(\mathrm{p}=0.001)$, and the grade of cancer $(p=0.041)$, in such a way that TSP-1 being more frequently detected in non-mucinous carcinomas and moderately differentiated tumors.

Clinical studies of patients with CRC have investigated levels of TSP-1 and correlated it with angiogenesis [14, 15, 39]. This relationship was established based on a significant association between TSP-1 expression, VEGF expression and MVD count. Tumors from patients that expressed high levels of TSP-1 and had low MVD counts were more likely to exhibit a decrease in angiogenesis compared to control tissue. The inverse has also been documented; a decrease in TSP-1 expression was accompanied with high MVD counts which may contribute to an angiogenic phenotype [31, 39, 40]. In the present study, we failed to demonstrate any correlation between TSP-1 expression, MVD and VEGF expression. In our opinion the two markers are not necessarily linked to each other.

The most common genetic alteration in CRC is reported to be the loss of p53 tumor suppressor gene function. The p53 protein has various important functions in cellular integration, including cell growth control, response to DNA damage, checkpoint mechanisms during the cell cycle, regulation of transcription and control of genomic stability. It has been reported that loss of tumor suppressor gene expression and oncogene activation are both associated with the enhancement of angiogenesis [41, 42]. Mutant p53 has been reported to regulate VEGF-activating pathways, whereas after loss of wild-type p53, a decrease in angiogenesis inhibitors, such as thrombospondin-1 was observed [41-43]. The study of Tokunaga et al, showed that TSP-1 expression was inversely associated with p53 accumulation in colon cancer and they suggested that p53 accumulation is closely associated with angiogenic phenotype through TSP-1 expression in CRC [9]. We did not observe any relationship with p53, which may be related to the discrepancy between p53 immunostaining and mutation analysis. Positive immunostaining for p53 may not reflect a mutated status of the protein.

To our knowledge there are no reports concerning the correlation between TSP-1 expression and the ECM in CRC. Fibronectins may play an important role in vascular stroma formation because they are essential for the heart and blood vessel morphogenesis, to be chemotactic for endothelial cells, to modulate endothelial response to growth factors, and to promote the elongation of microvessels during angiogenesis in vitro [25]. An in situ hibridization study of human colon cancer tissues showed a positive correlation between FNmRNA expression and the depth of invasion as well as the frequency of lymph node metastases, suggesting that FN expression could be important for the remodeling process of neoplastic tissues during cancer development and progression [25]. In our study, FN expression was correlated with TSP-1 supporting our hypothesis that extracellular matrix components acting together in the development and progression of CRC. Tenascin is a glycoprotein component of the extracellular matrix, which seems to have competitive functions with fibronectin [23]. It can be speculated that this competitive relationship between these molecules is important for cellular functions [23]. Tenascin expression indicates 
an altered cell-extracellular matrix interaction that might facilitate epithelial tumor invasion [22]. Tenascin expression is highly upregulated during tumor invasion in many tumor types, including colorectal carcinomas [24]. In the present study we found a borderline relationship of TSP-1 expression with $\mathrm{TN}(\mathrm{p}=0.053)$. Another interesting finding in this report, is the relationship between TSP-1 with laminin $(p=0.0001)$, and type IV collagen $(p=0.004)$, suggesting the contribution of these glycoproteins in tumor stroma, remodeling the effect of these ECM products in colorectal vascularization.

TSP-1 expression has also been a predictor of tumor recurrence and overall survival $[42,43]$. In patients with primary CRC with low TSP-1 expression with or without detection of mp53 gene product are more likely to harbor lymph node metastasis than patients with higher expression [41]. Sutton et al, reported that TSP-1 is expressed differently in liver metastasis when compared with the primary tumor, possibly showing different modes of action such as facilitating tumor invasion rather than acting as antiangiogenic growth factor [16]. Maeda et al, concluded in their work, that patients with TSP-1 negative tumors had a significantly worse prognosis than those with TSP-1 positive tumors and the frequency of hepatic recurrence was significantly higher in patients with tumors that were TSP-1 negative [15]. In our study, the prognostic significance of TSP-1 expression in colorectal carcinomas in both tumor cells and tumor stroma in determining the risk of recurrence and progression with univariate and multivariate methods of analysis showed no statistically significant differences $(\mathrm{p}=0.072$ and $\mathrm{p}=0.076$ respectively).

Thrombospondin-1 expression has been extensively studied in the last years both as prognostic factor and as predictor of response to chemotherapy [44, 45]. 5- fluorouracil (5-FU) is a commonly used anticancer drug against CRC. It has been demonstrated that 5-FU-based drug, S-1 upregulated TSP-1 and down-regulated microvessel formation in colorectal cancer xenografts with inhibition of tumor growth without remarkable toxicity in mice. Similar results were obtained with the use of irinotecan (CPT-11), another chemotherapeutic agent. When TSP-1 was combined with CPT-11, a significant inhibition of tumor growth was observed without concomitant increase in toxicity [45].

\section{CONCLUSIONS}

Taking the above information into account, we can suggest that TSP-1 expression, although does not seem to be a direct anti-angiogenic factor, could be implicated in the remodeling of colorectal cancer tissue through the interactions with other ECM components. On the basis of available literature data and in the light of the results from the present study, it is not possible to draw any definitive conclusion about the role of TSP-1 in predicting the clinical outcome of patients with colorectal cancer. Even though it is not a diffuse custom among clinical researchers, future investigations should be carefully planned to achieve an adequate level of evidence that will allow the questions put forward to be answered.

\section{CONFLICT OF INTERSEST}

The authors confirm that this article content has no conflicts of interest.

\section{ACKNOWLEDGEMENTS}

None declared

\section{REFERENCES}

[1] Harpaz N, Saxena R. Large Intestine. In: Modern Surgical Pathology, 1st ed. Weidner N, Cote RJ, Weis Lm, Eds. Philadelphia; Sauders, 2003; pp. 749-852.

[2] Jemal A, Siegel R, Xu J, Ward E. Cancer statistics, 2010. CA Cancer J Clin 2010; 55: 74-108.

[3] Kraemer M, Wiratkapun S, Seow-Cheon F, Ho YH, Eu KW, Nyan D. Stratifying risk factors for follow-up: a comparison of recurrent and non recurrent colorectal cancer. Dis Colon Rectum 2001; 44: 815-21.

[4] Carmelit P, Jairo RK. Angiogenesis in cancer and other diseases. Nature 2000; 407: 249-57.

[5] Chen H, Herndon ME, Lawler J. The cell biology of thrombospondin-1. Matrix Biol 2000; 19: 597-614.

[6] de Fraipon F, Nicholson AC, Feige JJ, Van Meir EG. Thrombospondins and tumour angiogenesis. Mol Med 2001; 7: 401-7.

[7] Rak J, Klement G. Impact of oncogenes and tumor suppressor genes on deregulation of hemeostasis and angiogenesis in cancer. Cancer Metastasis Rev 2000; 19: 93-6.

[8] Dameron KM, Volpert OV, Tainsky MA, Bouck N. Control of angiogenesis in fibroblasts by $\mathrm{p} 53$ regulation of thrombospondin-1. Science 1994; 265: 1582-4.

[9] Tokunaga T, Nakamura M, Oshika Y, et al. Alterations in tumor suppressor gene p53 correlate with inhibition of thormbospondin-1 gene expression in colon cancer cells. Virchows Arch 1998; 433: 415-8.

[10] Vallbo C, Wang W, Damber JE. The expression of thrombospondin-1 in benign prostate, hyperplasia and prostatic intraepithelial neoplasia is decreased in prostate cancer. BJU Int 2004; 2 : 1339-43.

[11] Mascaux C, Martin B, Paesmans M, et al. Expression of thrombospondin in non-small cell lung cancer. Anticancer Res 2002; 22: 1273-8.

[12] Weinstar-Saslow DL, Zabrenetzky VS, Vanltoutte K, Frazier WA, Roberts DD, Steeg PS. Transfection of thrombospondin-1 complementary DNA into a human breast carcinoma cell lines reduces primary tumour growth, metastatic potential, and angiogenesis. Cancer Res 1994; 54: 6504-11.

[13] Ioachim E, Michael MC, Salmas M, et al. Thrombospondin-1 expression in urothelial carcinoma: prognostic significance and association with p53 alterations, tumour angiogenesis and extracellular matrix components. BMC Cancer 2006; 6: 140-7.

[14] Maeda K, Nishiguchi Y, Yashiro M, et al. Expression of vascular endothelial growth factor and Thrombospondin-1 in colorectal carcinoma. Int J Mol Med 2000; 5: 373-8.

[15] Maeda K, Nishiguchi Y, Kang SM, et al. Expression of thrombospondin-1 inversely correlated with tumour vascularity and haematogenous metastases in colon cancer. Oncol Rep 2001; 8: 7638.

[16] Sutton CD, O'Byrne K, Goddard JC, et al. Expression of thrombospondin-1 in resected colorectal liver metastases predicts poor prognosis. Clin Cancer Res 2005; 11: 6567-73.

[17] Ferrara N. Vascular endothelial growth factor. Eur J Cancer 2006; 32A: 2413-22.

[18] Bergers G, Benjamin LE. Tumorigenesis and angiogenic switch. Nat Rev Cancer 2003; 3: 401-10.

[19] Kaio E, Tanaka S, Kitadai Y, et al. Clinical significance of angiogenic factor expression at the deepest invasive site of advanced colorectal carcinoma. Oncology 2003; 64: 61-73.

[20] Galindo-Gallego M, Fernandez Acarero MJ, Sanz Ortega J, Aljanta A, Lopez Blaurdia C. Prognositc significance of microvascular counts in rectal carcinoma. Pathol Res Pract 2000; 196: 607-12.

[21] Iozzo RN. Tumour stroma as a regulator of neoplastic behaviour. Lab Invest 1995; 73: 157-60. 
[22] Gulubova M, Vlaykova T. Immunohistochemical aeessment of fibronectin and tenascin and their integrin receptors $\alpha 5 \beta 1$ and $\alpha 9 \beta 1$ in gastric and colorectal cancers with lymph node and liver metastasis. Acta Histochem 2006; 108: 25-35.

[23] Chiquet-Ehrisman R, Kalla P, Pearson CA, Beck K, Chiquet M. Tenascin interferes with fibronectin action. Cell 1988; 53: 383-90.

[24] Lundin M, Nordling S, Lundin L, Haglund C. Tenascin-C expression and its prognostic significance in colorectal cancer. Oncology 2007; 72: 403-9.

[25] Tanjore H, Kalhuri R. The role of type IV collagen and basement membranes in cancer progression and metastasis. Am J Pathol 2006; 168: 715-7.

[26] De Arcangelis A, Lefebvre O, Mechine-Neuville A, et al. Overexpression of laminin $\alpha 1$ chain in colonic cancer cells induces an increase in tumor growth. Int J Cancer 2001; 94: 44-53.

[27] Compton CC, Greene FL. The staging of colorectal cancer: 2004 and beyond. CA Cancer J Clin 2004; 54: 295-308.

[28] Hamilton SR, Honen A. Pathology and genetics tumors of digestive system. World Health Organization-Classification of tumors. Lyon; IARC, Press 2000.

[29] Mitselou A, Skoufi U, Tsimogiannis KE, et al. Association of syndecan-1 with angiogenesis-related markers, extracellular matrix components, and clinicopathological features in colorectal carcinoma. Anticancer Res 2012; 32: 3977-86.

[30] Mitselou A, Ioachim E, Skoufi U, et al. Predictive role of thymidine phosphorylase expression in patients with colorectal cancer and its association with angiogenesis-related proteins and extracellular matrix components. In Vivo 2012; 26: xx (in press).

[31] Tanaka K, Sonoo H, Kurebayashi J, et al. Inhibition of infiltration and angiogenesis by thrombospondin-1 in papillary thyroid carcinoma. Clin Cancer Res 2002; 8: 1125-31.

[32] Linderholm B, Karlsson E, Klaar S, et al. Thrombospondin-1 expression in relation to 553 status and VEGF expression in human breast cancers. Eur J Cancer 2004; 40: 2417-23.

[33] Hayashi K, Kurohiji T, Shirouzu K. Localization of thrombospondin in hepatocellular carcinoma. Hepatology 1997; 25: 56974 .

[34] Zhang J, Ito R, Oue N, et al. Expression of thrombospondin-1 is correlated with microvessel density in gastric carcinoma. Virchows Arch 2003; 442: 563-8.
[35] Kasper HU, Ebert M, Malfertheiner P, Roessner A, Kikpatrick CJ, Wolf HK. Expression of thrombospondin-1 in pancreatic carcinoma: correlation with microvessel density. Virchows Arch 2001; 438: $116-20$.

[36] Oshiba G, Kijima H, Himeno S, et al. Stromal thrombospondin-1 expression is correlated with progression of esophageal squamous cell carcinomas. Anticancer Res 1999; 19: 4375-8.

[37] Rice AJ, Steward MA, Quinn CM. Thrombospondin 1 protein expression relates to good prognostic indices in ductal carcinoma in situ of the breast. J Clin Pathol 2002; 55: 921-5.

[38] Ioachim E, Damala K, Tsanou E, et al. Thrombospondin-1 expression in breast cancer: prognostic significance and association with p53 alterations, tumour angiogenesis and extracellular matrix components. Histol Histopathol 2012; 27: 209-16.

[39] Rojas A, Meherm S, Kim YH, et al. The aberrant methylation of TSP-1 suppresses TGF $\beta 1$ activation in colorectal cancer. Int J Cancer 2003; 123: 14-21.

[40] Kato E, Tanaka S, Oka S, et al. Clinical significance of thrombospondin-1 expression in relation to vascular endothelial growth factor and interleukin-10 expression at the deepest invasive tumor site of advanced colorectal cancer. Int J Oncol 2003; 23: 901-11.

[41] Sundaram P, Hultine S, Smith LM, et al. P53 responsive miR-194 inhibits thrombospondin-1 and promotes angiogenesis in colon cancers. Cancer Res 2011; 71: 7490-501.

[42] Bendardaf R, Buhmeida A, Hilska M, et al. VEGF-1 expression in colorectal cancer is associated with disease localization, stage, and long-term disease-specific survival. Anticancer Res 2008; 28: 3865-70.

[43] Iddings DM, Koda EA, Grewal SS, Parker R, Saha S, Rilchik A. Association of angiogenesis markers with lymph node metastasis in early colorectal cancer. Arch Surg 2007; 142: 738-45.

[44] Allegrini G, Goulette FA, Darnowski JW, Calabresi P. Thrombospondin-1 plus irinotecan: a novel antiangiogenicchemotherapeutic combination that inhibits the growth of advanced human colon xenografts in mice. Cancer Chemother Pharmacol 2004; 53: 261-6.

[45] Zhao HY, Ooyama A, Yamamoto M, et al. Molecular basis for the induction of an angiogenic inhibitor thrombospondin-1, by 5fluorouracil. Cancer Res 2008; 68(17): 7035-41. 